\title{
The effectiveness and cost effectiveness of telephone counselling and the nicotine patch in a state tobacco quitline
}

\author{
Jack F Hollis, Timothy A McAfee, Jeffrey L Fellows, Susan M Zbikowski, Michael Stark, Karen \\ Riedlinger
}

Tobacco Control 2007; 16(Suppl I):i53-i59. doi: 10.1136/tc.2006.019794

See end of article for authors' affiliations

Correspondence to:

Jack F Hollis, Center for Health Research, Kaiser Permanente Northwest, $3800 \mathrm{~N}$ Interstate Ave, Portland, OR 97227, USA; Jack.Hollis@kpchr.org

Received 28 December 2006 Accepted 20 August 2007

\begin{abstract}
Objectives: State and national tobacco quitlines have expanded rapidly and offer a range of services. We examined the effectiveness and cost effectiveness of offering callers single session versus multisession counselling, with or without free nicotine patches.

Methods: This $3 \times 2$ randomised trial included 4614 Oregon tobacco quitline callers and compared brief (one 15-minute call), moderate (one 30-minute call and a follow-up call) and intensive (five proactive calls) intervention protocols, with or without offers of free nicotine patches (nicotine replacement therapy, NRT). Blinded staff assessed tobacco use by phone at 12 months.

Results: Abstinence odds ratios were significant for moderate $(\mathrm{OR}=1.22, \mathrm{Cl}=1.01$ to 1.48$)$ and intensive $(\mathrm{OR}=1.29, \mathrm{Cl}=1.07$ to 1.56$)$ intervention, and for $\mathrm{NRT}(\mathrm{OR}=1.58, \mathrm{Cl}=1.35$ to 1.85$)$. Intent to treat quit rates were as follows: brief no NRT (12\%); brief NRT (17\%); moderate no NRT (14\%); moderate NRT (20\%); intensive no NRT (14\%); and intensive NRT (21\%). Relative to brief no NRT, the added costs for each additional quit was $\$ 2467$ for brief NRT, \$1912 for moderate no NRT, \$2109 for moderate NRT, \$2641 for intensive no NRT, and \$2112 for intensive NRT.

Conclusion: Offering free NRT and multisession telephone support within a state tobacco quitline led to higher quit rates, and similar costs per incremental quit, than less intensive protocols.
\end{abstract}

$\mathrm{T}$ he past decade has produced a dramatic nationwide dissemination of a new form of behavioural therapy. In 1999, only four states (Oregon, Arizona, California, and Massachusetts) provided centralised telephone support services (quitlines) and none offered free nicotine replacement therapy (NRT). By 2005, all states in the United States, all provinces of Canada, Australia, New Zealand and many countries in the European Union and elsewhere had established quitlines. ${ }^{1-3}$ Services range from information, counselling, and referral in a single call to multisession counselling with proactive followup. ${ }^{4-6}$ More than 18 states currently provide NRT to some or all callers. ${ }^{1}$

Cessation medications, including nicotine replacement, ${ }^{78}$ bupropion, ${ }^{9}$ and varenicline, ${ }^{10-12}$ all increase success rates. Proactive, multiple session telephone counselling ${ }^{713-16}$ also improves outcomes, and the efficacy of cessation quitlines has been confirmed in statewide programmes ${ }^{17-19}$ serving large and diverse populations. ${ }^{462021}$ The US clinical practice guideline for tobacco concluded that both medications and quitlines are effective. ${ }^{7}$ Proactive quitlines provide support over multiple contacts, but are more convenient than group counselling and are often provided free of charge. These features allow quitlines to attract more and more diverse, tobacco users than do group programmes. ${ }^{22}$ The efficacy and broad reach of quitlines create a potentially large population impact.

Few randomised trials have assessed the relative effectiveness and cost effectiveness of alternative quitline services. Borland ${ }^{23}$ and $\mathrm{Zhu}^{24}$ found that adding follow-up calls to an initial telephone counselling session increased one-year abstinence rates. Gilbert et al, ${ }^{25}$ however, found that five nonstructured, client led counselling calls offered no benefit over a single call. Swan et $a l_{,}{ }^{26}$ Zhu et $a l^{27}$ and McLeod $^{28}$ all found that repeated phone counselling increased the long term effectiveness of cessation medications, though three smaller studies found no benefit. ${ }^{29-31}$ Cummings reported that quit rates were higher for smokers who called a quitline during a period when free NRT patches were offered compared to those who called when patches were not available. ${ }^{32}$ A recent Cochrane review concluded that providing proactive counselling as adjunct to medications improved quit outcomes by $28 \%{ }^{15}{ }^{1632}$ This limited and conflicting evidence base (along with funding limitations) may explain why few quitlines routinely offer multisession counselling and pharmacological aids to most callers.

The potential impact of this national and international investment in quitlines raises several key questions. Do multiple and/or longer call protocols improve outcomes? Are more intensive protocols cost effective compared to a single brief call? Does offering free NRT increase quitline success rates in a cost effective manner? This study provides policy relevant evidence about effectiveness and cost effectiveness of brief, moderate and multisession telephone counselling, with or without NRT therapy, within a real world quitline setting. ${ }^{33}$

\section{METHODS}

\section{Setting and design}

This study was conducted within the ongoing operations of the Oregon tobacco quitline (OTQL). Oregon used mass media campaigns, direct mailings to select populations (for example, Medicaid) and encouragement to physicians and health plans to recruit tobacco users to the OTQL. OTQL telephone counselling services were provided through a contract with Free \& Clear, Inc, a Seattle based service provider. Eligible and consenting callers were randomly assigned to one of six interventions in a 3 (behavioural) $\times 2(\mathrm{NRT})$ design that compared brief, moderate and intensive telephone counselling, with or without an offer of free NRT patches.

Abbreviations: ICER, incremental cost effectiveness ratios; NRT, nicotine replacement therapy; OTQL, Oregon tobacco quitline 


\section{Screening and randomisation}

Between 26 January 2001 and 20 January 2003, registration staff described the study to quitline callers and assessed their interest and eligibility. Callers who were not eligible or interested in the study received standard OTQL services. Eligible callers were Oregon residents who were 18 years of age or older, spoke English or Spanish, smoked five or more cigarettes per day over the past six months and were planning to quit within the next month (or had quit within the preceding seven days). Callers were excluded if their health plan provided multisession telephone counselling through Free and Clear, Inc, as a covered benefit, or if they had medical conditions that would contraindicate patch use, including pregnancy, breast feeding, plans to become pregnant or a history of heart attack within the preceding month. Initially, callers with no health insurance were excluded because state policy provided free access to the multisession phone counselling with free NRT patches. When this state benefit ended midway through the recruitment period, uninsured callers became eligible for the study.

Eligible and interested callers next spoke to a tobacco intervention specialist who confirmed the caller's understanding of the study, answered questions and confirmed and documented informed consent. Consent included agreeing to randomisation and to considering offers of additional free services, such as phone support and/or NRT, without committing to actually use any services offered. Participants also consented to six-month and 12-month follow-up assessments. The Kaiser Permanente Northwest institutional review board and Public Health institutional review board for the Oregon Department of Health Services approved this study. Once the consent was confirmed, a computer algorithm randomly assigned participants.

\section{Intervention}

Staffing, training and quality control

Experienced telephone tobacco counsellors provided the interventions after receiving additional theoretical and practical training in motivational interviewing. Training focused on how to adhere closely to the different intervention protocols using computer driven scripting. We taped calls for quality assurance monitoring and rated counsellors regularly on adherence to key elements of each protocol.

\section{Community and health plan referral information}

All participants were offered referral information for their health plan and county of residence, including phone numbers, contact names, costs, co-pays and programme descriptions.

\section{Cessation and mail materials}

All callers were mailed a "quit kit" that included a cessation booklet (Stop Smoking, Smoke-Free for Life) and, if applicable, other materials on stress management, smokeless tobacco, secondhand smoke, pharmacotherapy and social support. Participants in the intensive cells who accepted additional counselling calls also received a personalised letter with their quit plan and a 60-page cessation support booklet. Counselling and written materials were available in English or Spanish.

\section{Brief counselling}

Brief counselling included one 15-minute call. Specialists used a brief negotiation model ${ }^{34-36}$ to communicate caring, motivate change and provide information on recommended quitting strategies. Callers were encouraged to set a quit date, ask for support from friends and family, remove cigarettes from home, car and workplace, anticipate challenges and review previous quit attempts.

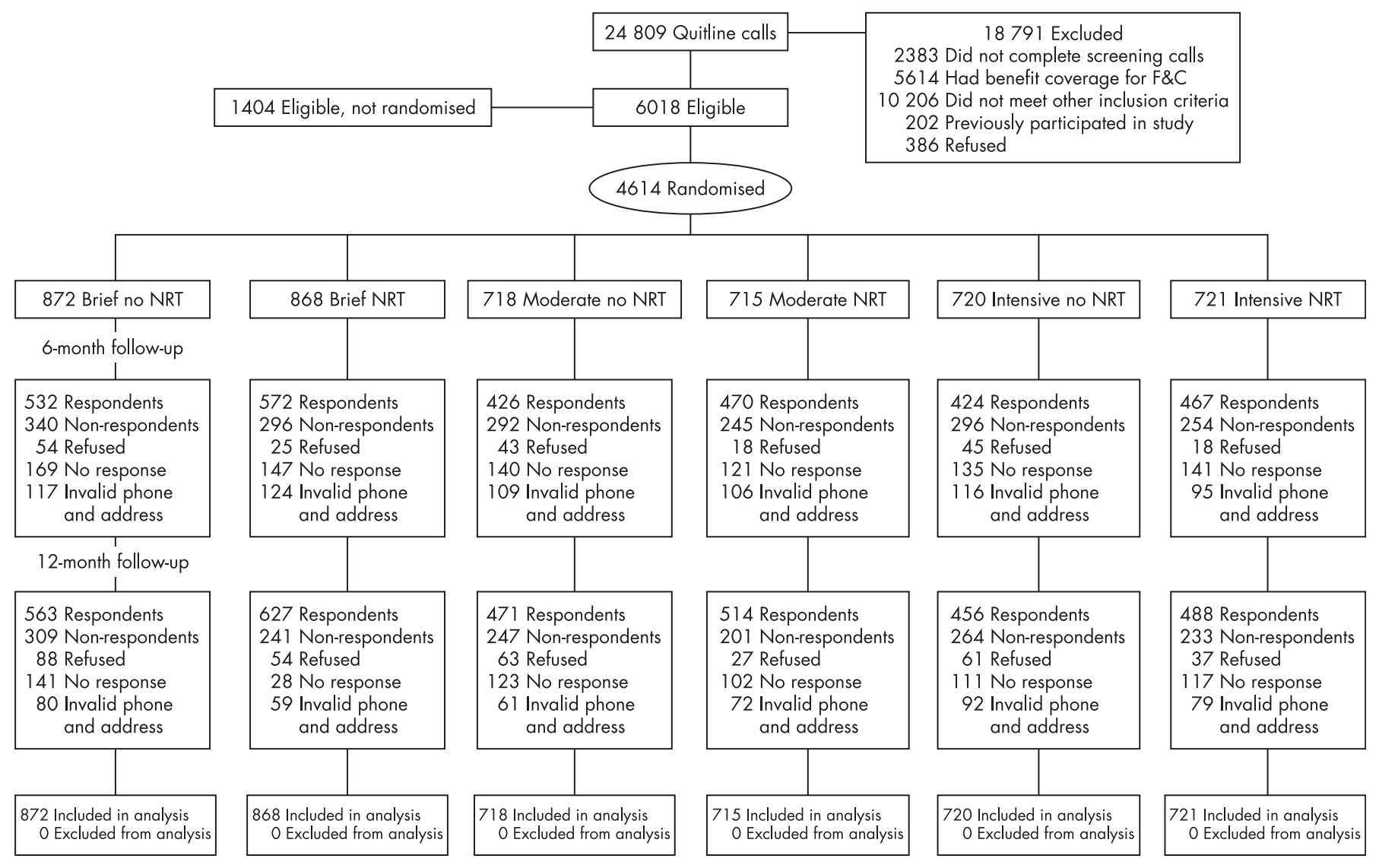

Figure 1 Study flow diagram. 


\section{Moderate counselling}

Moderate counselling included an initial 40-minute counselling session based on the motivational interviewing model ${ }^{36}$ and a brief follow-up call one to two weeks later to support the quit plan and encourage use of health plan or community services and mailed cessation materials.

\section{Intensive counselling}

Intensive counselling included an initial 30-minute to 40minute counselling session plus an offer of up to four additional telephone support calls over a three-month period. Each follow-up call incorporated motivational interviewing techniques, stage assessment and relapse prevention, as needed.

\section{Nicotine replacement offer}

All study participants received information about pharmacotherapy options. Participants randomised to the offer of free NRT patches received a more in-depth NRT assessment. Callers with NRT related medical concerns or possible contraindications were referred to their physicians. Specialists reviewed proper NRT use and signs of overdose or inadequate dose and instructed NRT users to call back with questions or concerns. Dosage and duration recommendations were based on FDA guidelines. Participants initially received a five-week supply and then placed a toll-free call to request a refill for the final three weeks.

\section{Follow-up assessments}

Trained assessors blinded to treatment condition conducted follow-up assessments by phone six months and 12 months after randomisation. At each follow-up, assessors made at least 10 attempts to reach participants during a range of day, evening and weekend hours. Non-respondents were sent a shortened self administered version of the questionnaire with a \$20 incentive for returning a completed survey. If the participant's phone and address information was no longer valid, assessors used collateral contacts, updated phone directories, reverse phone directories, web based person locators and DMV records to update information.

\section{Primary outcome measure}

The primary outcome measure was the proportion of participants reporting abstinence from all forms of tobacco for 30 days or more at the 12-month follow-up after randomisation. The 30-day point prevalence measure is a more stable measure than 7-day point prevalence and was chosen over a continuous abstinence measure because many successful long-term quitters (1) take some period of time before they set an initial quit date; and/or (2) report slips during the first few months after quitting. Those who refused or were lost to follow-up were coded as continued tobacco users.

\section{Statistical analysis}

This study was a randomised, parallel group design, with six treatment cells in a $2 \times 3$ factorial design. The sample sizes for the two brief intervention cells were enriched to provide enhanced power to detect the effect of the NRT offer with minimal behavioural support. The sample sizes per cell were easily large enough to permit the usual normal approximation to the sampling distribution of the binomial distribution. Power was adequate $(0.80$, alpha $=0.05)$ to detect a difference in abstinence of 0.04 (for example, 0.08 versus 0.12 ). We used multiple logistic regression to compare the moderate and intensive interventions to brief intervention, the NRT offer to no NRT offer and an NRT offer with the moderate compared to intensive arms.
The primary end point was smoking cessation at 12 months using traditional intent to treat assumptions. We also conducted two secondary analyses using multiple imputation procedures $^{37}$ to account for missing outcome data. Firstly, we used baseline age, sex, income, education, presence of other smokers in the household and smoking rate to create five imputed datasets that were then averaged using the Solas program..$^{38}$ Secondly, we used the same baseline variables to impute outcomes only for those with invalid phone or address information at follow-up and assumed that those who actively refused follow-up or did not respond to voice mail or mailed surveys (that is, passive refusals) were smoking.

\section{Cost effectiveness analysis}

We estimated the incremental cost effectiveness of each intervention compared to brief counselling no NRT from a state programme perspective. Costs were based on what was actually provided to each individual rather than what was intended according to protocol. We estimated total training costs and the differential delivery costs for each intervention, including all labour, facility space and supplies (for example, quit kits). Labour costs included salary and benefits for interventionists, supervisory staff (including medical oversight) and administrative staff. Interventionist time included the actual time spent with individual participants, off-line preparation, training and other administrative functions. Contact time with participants included screening and enrolment, intervention delivery calls and ad hoc calls. Facility space, technical and administrative support and office supplies were estimated using the indirect cost rate for the intervention delivery organisation. NRT costs were calculated as the programme price for actual shipments plus postage, as obtained from pharmacy records. Though Nicoderm CQ was provided free by GlaxoSmithKline, our analyses used the usual NRT costs paid by Free \& Clear, Inc. We used ordinary least squares regression to calculate mean total programme costs and standard deviations for participants in each intervention. We calculated incremental costs per additional quit (that is, incremental cost effectiveness ratios, ICER) for each intervention compared to brief no NRT. Ranges for each ICER were estimated using the 12-month abstinence rates. All costs were translated into 2004 dollars using the medical care component of the consumer price index. ${ }^{39}$

\section{RESULTS}

\section{Recruitment and participants}

The OTQL received 24809 in-coming calls between 26 January 2001 and 20 January 2003. Callers reported hearing about the OTQL from TV commercials (29\%); TV news (13\%); radio, newspapers, or billboards $(9 \%)$; brochures, fliers, or mailers (6\%); family or friends (19\%); medical care providers $(14 \%)$; dental care providers ( $1 \%)$; and other sources (or did not recall) $(5 \%)$.

Of all callers, $6018(24 \%)$ met study eligibility requirements. Callers were ineligible if they: (1) did not complete the screening call (13\% of ineligibles); (2) were not eligible or interested in quitline services (for example, proxy, self help, or out of state callers) (12\%); (3) previously participated in the study $(1 \%) ;(4)$ had free access to multisession counselling as a covered benefit $(30 \%)$; (5) were less than 18 years of age, smoked fewer than five cigarettes per day, were pregnant or planning to become pregnant, had a myocardial infarction in past 30 days or did not speak either English or Spanish (12\%); (6) were not planning to quit within 30 days (4\%); and (7) were not interested in participating in research $(28 \%)$. Of the 6018 eligible callers, $4614(77 \%)$ consented and were randomised (fig 1). 
Table 1 Baseline characteristics

\begin{tabular}{|c|c|c|c|c|c|c|c|}
\hline \multirow[b]{2}{*}{ Characteristics } & \multicolumn{3}{|c|}{ No NRT offer } & \multicolumn{4}{|l|}{ NRT offer } \\
\hline & $\begin{array}{l}\text { Brief } \\
(n=872)\end{array}$ & $\begin{array}{l}\text { Moderate } \\
(n=718)\end{array}$ & $\begin{array}{l}\text { Intensive } \\
(n=720)\end{array}$ & $\begin{array}{l}\text { Brief } \\
(n=868)\end{array}$ & $\begin{array}{l}\text { Moderate } \\
(n=715)\end{array}$ & $\begin{array}{l}\text { Intensive } \\
(n=721)\end{array}$ & p Value \\
\hline Female & 59.5 & 59.2 & 62.2 & 60.3 & 59.2 & 59.0 & 0.81 \\
\hline Age (years), mean (SD) & $41.1(13.1)$ & $41.4(13.1)$ & 40.8 (12.7) & $41.0(13.4)$ & $41.4(13.0)$ & 40.5 (13.8) & 0.77 \\
\hline Some college (\%) & 51.6 & 51.7 & 47.6 & 52.2 & 53.7 & 51.3 & 0.32 \\
\hline White (\%) & 89.6 & 92.6 & 89.3 & 91.8 & 90.2 & 88.9 & 0.08 \\
\hline Hispanic (\%) & 6.0 & 3.3 & 6.0 & 5.7 & 5.3 & 4.7 & 0.18 \\
\hline Spanish speaker (\%) & 0.1 & 0 & 0.4 & 0.5 & 0 & 0.3 & NA \\
\hline Married/partnered (\%) & 43.0 & 46.2 & 42.6 & 43.1 & 43.5 & 42.6 & 0.73 \\
\hline Medical coverage (\%) & 70.2 & 73.7 & 74.6 & 72.0 & 75.4 & 71.7 & 0.18 \\
\hline Cigarettes, mean (SD) & $21.9(10.5)$ & $21.8(10.2)$ & 21.5 (11.2) & $21.8(10.7)$ & $22.0(10.7)$ & $21.6(10.7)$ & 0.96 \\
\hline Other smoker in home (\%) & 40.3 & 47.1 & 43.6 & 45.5 & 43.9 & 43.0 & 0.12 \\
\hline
\end{tabular}

Participants were $60 \%$ female, middle aged and half had some college education (table 1 ). Race and ethnicity distributions reflected the Oregon population and only $1 \%$ of participants were primarily Spanish speakers. Less than half were married or partnered and most reported some form of health insurance. Most were moderate to heavy smokers and nearly half had other smokers in the home. By design, all participants were planning to quit within the next 30 days. About 3\% $(\mathrm{n}=149)$ had quit smoking less than 7 days before randomisation, of whom about half $(n=64)$ quit within 24 hours of the call. Participants rated themselves high on readiness to quit and somewhat confident (that is, 7) they could quit. Participants' reasons for quitting included health $(43 \%)$, expense $(11 \%)$, setting an example for children $(10 \%)$, smell (5\%), family/friends (4\%), the effects of smoking on nonsmokers $(3 \%)$, addiction $(2 \%)$, illness or death of a family member or friend (1\%) and a doctor's advice (1\%). There were no significant differences between the six groups on baseline characteristics (table 1).

\section{Intervention delivery and process measures}

The number of calls increased from a mean of 1.0 for the brief no NRT condition to 2.9 for intensive NRT (table 2). In both the NRT and no NRT conditions, the mean number of calls completed were significantly greater in the intensive compared to either the moderate and brief cells (all p values $<0.0001$ ), though the absolute differences were modest. Within the two intensive cells, the percentage of callers completing various number of follow-up calls in the NRT and no NRT conditions were: $0-1$ call $(24 \%$ and $31 \%), 2$ calls $(21 \%$ and $23 \%), 3$ calls ( $18 \%$ and $18 \%$ ) and 4 or more calls (36\% and $28 \%$ ). In each behavioural arm, participants offered NRT completed somewhat more calls than those not offered NRT (for example, to request refills and/or ask questions) (all p values $<0.0001$ ). The total telephone contact time (including about 10 minutes for baseline screening and consent) increased across conditions, as expected, with more contact time in the three NRT cells versus the comparable no NRT cells. Of those offered NRT, $80 \%$ accepted the first 5-week regimen and $25 \%-28 \%$ requested a second 3 -week refill and there were no differences across the three levels of behavioural intensity $(p=0.862)$.

\section{Follow-up assessments}

Data on tobacco use status were obtained for $67 \%$ of randomised participants at 6 months and 69\% at 12 months.

\section{Satisfaction with quitline services}

Participants offered moderate or intensive support, compared to brief (table 2), were more satisfied with the quitline and more likely to report that they received the "right amount of contact." Within each behavioural arm, those offered NRT were more likely to be satisfied and report they received the right amount of contact.

\section{Tobacco cessation}

Table 3 presents end point data assuming those lost to followup were smoking. At six months, abstinence rates ranged from a low of $10 \%$ for brief no NRT to a high of $24 \%$ for intensive NRT. Logistic regression odds ratios for cessation were significantly higher for NRT versus no NRT $(\mathrm{OR}=2.04$; CI: 1.73 to 2.41 ) and for both the moderate ( $\mathrm{OR}=1.22$; $\mathrm{CI}$ : 1.002 to 1.49 ) and intensive ( $\mathrm{OR}=1.48$; $\mathrm{CI}$ : 1.22 to 1.79 ) interventions, relative to brief no NRT. By 12 months, differences had narrowed (range $12 \%$ to $21 \%$ ), but treatment effects remained statistically significant for the NRT $(\mathrm{OR}=1.58$; $\mathrm{CI}$ : 1.35 to $1.85)$, moderate $(\mathrm{OR}=1.22 ; \mathrm{CI}: 1.01$ to 1.48$)$ and intensive $(\mathrm{OR}=1.29$; CI: 1.07 to 1.56$)$ comparisons. Neither the moderate $\times$ NRT nor the intensive $\times$ NRT interaction was significant at

Table 2 Services used and caller satisfaction

\begin{tabular}{|c|c|c|c|c|c|c|c|}
\hline \multirow[b]{2}{*}{ Characteristics } & \multicolumn{3}{|c|}{ No NRT offer } & \multicolumn{4}{|l|}{ NRT offer } \\
\hline & $\begin{array}{l}\text { Brief } \\
(n=872)\end{array}$ & $\begin{array}{l}\text { Moderate } \\
(n=718)\end{array}$ & $\begin{array}{l}\text { Intensive } \\
(\mathrm{n}=\mathbf{7 2 0})\end{array}$ & $\begin{array}{l}\text { Brief } \\
(n=868)\end{array}$ & $\begin{array}{l}\text { Moderate } \\
(n=715)\end{array}$ & $\begin{array}{l}\text { Intensive } \\
(\mathrm{n}=721)\end{array}$ & p Value \\
\hline Counselling sessions, mean (SD) & $1.0(0.3)$ & $1.7(0.7)$ & $2.5(1.5)$ & $1.3(0.7)$ & $2.0(0.9)$ & $2.9(1.6)$ & $<0.0001$ \\
\hline Contact time (minutes), mean (SD) & $20.1(9.9)$ & $41.3(16.8)$ & $53.6(28.7)$ & $23.9(13.0)$ & $47.1(20.7)$ & $60.6(29.7)$ & $<0.0001$ \\
\hline \multicolumn{8}{|l|}{ NRT accepted } \\
\hline 1st shipment (\%) & NA & NA & NA & 79.6 & 80.3 & 80.0 & 0.94 \\
\hline 2nd shipment (\%) & & & & 25.2 & 27.6 & 27.2 & 0.52 \\
\hline Satisfied with quitline $(\%)^{*} \dagger$ & 53.9 & 66.8 & 80.7 & 82.2 & 88.3 & 92.5 & $<0.0001$ \\
\hline "Right amount of contact" (\%)* $\ddagger$ & 34.5 & 47.8 & 64.9 & 48.7 & 58.2 & 71.6 & $<0.0001$ \\
\hline
\end{tabular}

*Based on a 4-point item collected at the six-month follow-up.

$\dagger n=2753$, response rate is $60 \%$.

fn $=2874$, response rate is $62 \%$. 
Table 3 Smoking cessation and cost effectiveness

\begin{tabular}{|c|c|c|c|c|c|c|c|}
\hline \multirow[b]{2}{*}{ Characteristics } & \multicolumn{3}{|c|}{ No NRT offer } & \multicolumn{4}{|l|}{ NRT offer } \\
\hline & $\begin{array}{l}\text { Brief } \\
(n=872)\end{array}$ & $\begin{array}{l}\text { Moderate } \\
(n=718)\end{array}$ & $\begin{array}{l}\text { Intensive } \\
(n=720)\end{array}$ & $\begin{array}{l}\text { Brief } \\
(n=868)\end{array}$ & $\begin{array}{l}\text { Moderate } \\
(n=715)\end{array}$ & $\begin{array}{l}\text { Intensive } \\
(n=721)\end{array}$ & p Value \\
\hline $\begin{array}{l}\text { Abstinence* } 6 \text { months (\%) }^{*} \\
\text { Abstinence }^{*} 12 \text { months (\%) } \\
\text { Cost/participant (SD), 2004\$ } \\
\text { Incremental cost/quitt (range), } \\
2004 \$\end{array}$ & $\begin{array}{l}10.2 \\
11.7 \\
\$ 67(\$ 20) \\
\text { NA }\end{array}$ & $\begin{array}{l}10.7 \\
13.8 \\
\$ 107(\$ 33) \\
\$ 1912 \\
(\$ 2551-\$ 1273)\end{array}$ & $\begin{array}{l}13.1 \\
14.3 \\
\$ 132(\$ 57) \\
\$ 2640 \\
(\$ 4120-\$ 1161)\end{array}$ & $\begin{array}{l}16.8 \\
17.1 \\
\$ 193(\$ 79) \\
\$ 2467 \\
(\$ 3622-\$ 1311)\end{array}$ & $\begin{array}{l}21.3 \\
20.1 \\
\$ 242(\$ 92) \\
\$ 2109 \\
(\$ 2980-\$ 1239)\end{array}$ & $\begin{array}{l}24.3 \\
21.2 \\
\$ 268(\$ 99) \\
\$ 2112 \\
(\$ 2946-\$ 1278)\end{array}$ & $\begin{array}{l}<0.0001 \\
<0.0001 \\
<0.0001 \\
\text { NA }\end{array}$ \\
\hline
\end{tabular}

*Abstinent from all forms of tobacco for 30 days or more at follow-up.

†Incremental cost per additional quit relative to brief/no NRT arm. Ranges calculated using standard deviations and 12-month abstinence.

either the six-month or 12-month assessments, which indicates that NRT produced a similar proportional increase in quit rates across the three levels of behavioural intensity.

\section{Multiple imputation for missing values}

After replacing all missing outcomes using multiple imputation procedures, the odds ratio for NRT (OR $=1.30$; CI: 1.07 to 1.56 ) remained significant, but substantially smaller than with the intent to treat assumptions above. The odds ratio for the intensive intervention remained significant and similar to that of the intent to treat approach (OR = 1.28; CI: 1.05 to 1.55$)$. The odds ratio for the moderate intervention, however, was lower and no longer significant ( $\mathrm{OR}=1.13$; $\mathrm{CI}$ : 0.95 to 1.35 ).

We next used multiple imputation to predict outcomes only for those with no valid contact information at the time of follow-up and counted those who actively or passively refused follow-up as smokers. With this more conservative approach, the odds ratios and confidence intervals for the NRT $(\mathrm{OR}=1.51$; $\mathrm{CI}: 1.29$ to 1.77$)$, intensive $(\mathrm{OR}=1.34$; $\mathrm{CI}$ : 1.11 to 1.61) and moderate (OR $=1.23$; CI: 1.01 to 1.49 ) comparisons closely matched those seen with the intent to treat approach.

\section{Cost and cost effectiveness}

The average programme cost per participant (excluding media costs) for the actual services provided ranged from a low of $\$ 67$ (SD \$20) for brief no NRT to a high of \$268 (SD \$99) for intensive NRT $(p<0.001)$ (table 3$)$. Duncan's multiple range test confirmed that all intervention means were statistically different from each other $(p<0.001)$. The added costs per incremental quit (compared to brief no NRT) were lowest and similar for the moderate no NRT, moderate NRT and intensive NRT conditions.

\section{DISCUSSION}

This study is the largest to date of the effectiveness and cost effectiveness of alternative tobacco quitline services and policies. One-year abstinence rates increased when quitline callers were offered: (1) free NRT patches shipped by mail; and/ or (2) more intensive counselling with follow-up calls. These effects for NRT and follow-up counselling calls are consistent with meta-analyses for research conducted in other settings. ${ }^{781516}$ Callers were also more satisfied with the quitline when they were offered NRT and/or additional follow-up counselling support. Customer satisfaction is important because of its relevance to word of mouth advertising and because policy makers often use this measure to evaluate the performance of quitlines.

The added costs of additional counselling and NRT were offset by their increased effectiveness and all conditions were highly cost effective enhancements to brief no NRT. While the cost per incremental quit was about $\$ 200$ less for moderate no NRT (\$1912) compared to either moderate NRT (\$2109) or intensive NRT (\$2112), moderate no NRT yielded a lower quit rate ( $14 \%$ vs $20 \%$ and $21 \%$, respectively). Overall, the differences in incremental cost effectiveness across the five enhanced treatment conditions were modest and policy makers should focus more on other important attributes of the interventions, such as overall quit rates achieved, client satisfaction and their potential to attract more smokers to quitlines. Others have found that short term offers of NRT resulted in marked spikes in quitline calls. ${ }^{40-44}$ If this effect persists, states could choose to reduce advertising and instead provide more intensive and effective cessation services.

The literature generally supports a dose-response relation between level of service provision and successful quitting, but our primary intent to treat analyses found that the difference between the moderate (that is, two-call) and intensive (up to five-call) protocols was modest. This finding was probably due to the small difference in the number of calls actually completed in the moderate versus intensive conditions (1.7-2.0 vs 2.5-2.9 calls completed). This small difference is surprising because, with a similar quitline protocol in a non-research context, over 48000 callers completed an average of 3.8 calls. ${ }^{45}$ Programme planners should do all they can to encourage callers to complete the full regimen of calls (for example, provide incentives and review counselling protocols to see if language needs to be changed to more strongly emphasise the importance of completing the entire protocol).

Our response rate at 12 months was modest (69\%), though fairly typical for low contact studies in community settings. Our primary outcome assumed that those with missing outcomes were still smoking. Because controversy remains about how to handle missing data in smoking trials, ${ }^{37}{ }^{46}$ we compared our primary intent to treat method to two different multiple imputation approaches. When we used multiple imputation methods to replace all missing outcomes, we found that the effect for the intensive intervention remained strong, the odds ratio for NRT was substantially reduced (though still significant) and the effect for the moderate intervention became nonsignificant. Multiple imputation strategies, however, are considered appropriate only when "missingness" is unrelated to outcome. This assumption may be more appropriate for those with invalid phone and address information than for those who chose not to respond to repeated messages and mailings. When we imputed values only for those with invalid phone and address information and counted "passive refusers" as smokers, results were similar to our primary intent to treat approach.

Strengths of this study include the large sample size and the opportunity to compare the effects of six alternative treatment regimens among the large portion (77\%) of eligible OTQL callers who consented. The high recruitment rate and naturalistic context of the study increase the external validity and policy relevance of the findings. We tested the range of services typically offered by state quitlines and provide data by which to judge the effectiveness and cost effectiveness of alternative approaches. 


\section{What this paper adds}

- Several large studies, a recent Cochrane review and the US clinical practice guideline for tobacco have all concluded that telephone quitlines increase quit rates. Because telephone quitlines are convenient to access and are often provided free of charge, they have the potential to serve a broader and more diverse group of tobacco users than traditional group programmes. Quitlines vary widely in terms of the services they offer, however, and few routinely offer multisession counselling and pharmacological aids to most callers. Randomised trials of the relative effectiveness and cost effectiveness of different protocols are limited.

- This randomised trial showed that one-year abstinence rates increased when quitline callers were offered: (1) free NRT patches shipped by mail; and/or (2) more intensive counselling with follow-up calls. Callers were also more satisfied with the quitline when they were offered NRT and/or additional support. The added costs of additional counselling and NRT were offset by their increased effectiveness and all conditions were highly cost effective enhancements to brief counselling without NRT.

A potential limitation is that our outcome relied on self reported abstinence. Although biochemical confirmation of abstinence is appropriate and necessary in some intervention settings, we $\mathrm{e}^{47}$ and others ${ }^{48-50}$ have argued against the need for biochemical validation in adult population based studies with no face to face contact. In these settings, the proportion of self reported quitters who test positive for cotinine is low and generally equal for treatment and control subjects; ${ }^{47}$ and the compliance with saliva collection procedures is poor $(50-60 \%$ at best), especially across a large geographic area like Oregon.

Another potential limitation is that we did not obtain quit data beyond one year. Because differences in quit rates converged somewhat between six months and 12 months, it is possible that treatment effects would have diminished further by two years and beyond. Quit rates could also diverge, as has been seen in some studies, ${ }^{26}$ owing to repeated quit attempts in treatment groups. The reduced effect size between six months and 12 months also suggests that we need to study increasing booster sessions or repeating the intervention for those who relapse. A recent VA study suggests almost two thirds of relapsers are interested in trying again with behavioural and pharmacological support in the next 30 days. ${ }^{51}$ Another limitation is that we could not use a placebo NRT in this effectiveness trial of a politically sensitive statewide service and thus the NRT effect may, in part, be due to expectancy effects. Also, effect sizes may have been reduced by contamination if some controls acquired NRT on their own or if some subjects who were mailed NRT did not use it. From a policy perspective, however, state decision makers may care less about the mechanisms and more about the total impact (and costs) of policies that include an NRT offer versus no offer.

The Department of Health and Human Services in collaboration with the states has created a national network of quitlines ${ }^{4}$ that can route anyone in the United States who calls a single number (1-800-QUITNOW) to their local state quitline. Health plans and employers are increasingly promoting similar services, sometimes coordinating closely with the state-level efforts. $^{52}$ As a result, many physicians are routinely referring interested patients to quitlines for more extensive tobacco cessation support than can be offered during a short office visit. ${ }^{53}$ Our data show that offering follow-up counselling and medications through a quitline cost effectively increased patient satisfaction and doubled the overall quit rate.

\section{ACKNOWLEDGEMENTS}

We thank Kathy Easter, Kathy Mount, Anne Perez and Miriam Philby for their help in coordinating study activities, Nancy Clarke for her invaluable advice and support throughout the study and the many telephone counsellors who served so many Oregon quitline callers.

\section{Authors' affiliations}

Jack F Hollis, Jeffrey L Fellows, Karen Riedlinger, Center for Health Research, Kaiser Permanente Northwest, Portland, OR, USA

Timothy A McAfee, Susan M Zbikowski, Free \& Clear, Inc, Seattle, WA, USA Michael Stark, Oregon Department of Human Services, Portland, OR, USA

Funding: This work was supported by the National Cancer Institute Igrant R01 CA86242), and we want to thank GlaxoSmithKline for supplying the nicotine patches used in the study.

Competing interests: JFH, JLF and KR have no competing interests. TAMCA and SMZ are with Free \& Clear, Inc, which is a for-profit company providing telephone counselling services.

\section{REFERENCES}

1 Cummins SF, Bailey L, Campbell S, et al. Tobacco cessation quitlines in North America: a descriptive study. Tob Control 2007;16(Suppl I):i9-15.

2 Miller CL, Wakefield M, Roberts L. Uptake and effectiveness of the Australian telephone Quitline service in the context of a mass media campaign. Tob Control 2003; 12(Suppl 2):ii53-8.

3 Wilson N, Grigg M, Graham L, et al. The effectiveness of television advertising campaigns on generating calls to a national quitline by Maori. Tob Control 2005;14:284-6.

4 Fiore MC, Croyle RT, Curry SJ, et al. Preventing 3 million premature deaths and helping 5 million smokers quit: a national action plan for tobacco cessation. Am J Public Health 2004;94:205-10.

5 Ossip-Klein DJ, Mclntosh S. Quitlines in North America: evidence base and applications. Am J Med Sci 2003;326:201-5.

6 Centers for Disease Control and Prevention. Telephone quitlines: a resource for development, implementation, and evaluation. Atlanta, GA: US Department of Health and Human Services, Centers for Disease Control and Prevention, National Center for Chronic Disease Prevention and Health Promotion, Office on Smoking and Health, 2004.

7 Fiore MC, Bailey WC, Cohen SJ, et al. Treating tobacco use and dependence: a clinical practice guideline. Rockville, MD: United States Department of Health and Human Services, 2000.

8 Hughes JR, Shiffman S, Callas P, et al. A meta-analysis of the efficacy of over-thecounter nicotine replacement. Tob Control 2003;12:21-7.

9 Hughes J, Stead L, Lancaster T. Antidepressants for smoking cessation. Cochrane Database Syst Rev 2004;CD000031.

10 Gonzales D, Rennard SI, Nides M, et al. Varenicline, an alpha4beta2 nicotinic acetylcholine receptor partial agonist, vs sustained-release bupropion and placebo for smoking cessation: a randomized controlled trial. JAMA 2006;296:47-55.

11 Jorenby DE, Hays JT, Rigotti NA, et al. Efficacy of varenicline, an alpha4beta2 nicotinic acetylcholine receptor partial agonist, vs placebo or sustained-release bupropion for smoking cessation: a randomized controlled trial. JAMA 2006;296:56-63.

12 Tonstad S, Tonnesen P, Hajek P, et al. Effect of maintenance therapy with varenicline on smoking cessation: a randomized controlled trial. JAMA 2006;296:64-71.

13 Lichtenstein E, Glasgow RE, Lando HA, et al. Telephone counseling for smoking cessation: rationales and meta-analytic review of evidence. Health Educ Res 1996;11:243-57.

14 Hopkins DP, Briss PA, Ricard CJ, et al. Reviews of evidence regarding interventions to reduce tobacco use and exposure to environmental tobacco smoke. Am J Prev Med 2001;20:16-66.

15 Stead LF, Perera R, Lancaster T. A systematic review of interventions for smokers who contact quitlines. Tob Control 2007;16(Suppl I):3-8.

16 Stead LF, Lancaster T, Perera R. Telephone counseling for smoking cessation. Cochrane Database Syst Rev 2006;19.

17 Borland R, Segan CJ, Livingston PM, et al. The effectiveness of callback counseling for smoking cessation: a randomized trial. Addiction 2001; 96:881-9.

18 Zhu SH, Anderson CM, Tedeschi GJ, et al. Evidence of real-world effectiveness of a telephone quitline for smokers. N Engl J Med 2002;347:1087-93.

19 McAlister AL, Rabius V, Geiger A, et al. Telephone assistance for smoking cessation: one year cost effectiveness estimations. Tob Control 2004;13:85-6.

20 Smith PM, Cameron R, McDonald PW, et al. Telephone counseling for population-based smoking cessation. Am J Health Behav 2004;28:231-41. 
21 Zhu SH, Anderson CM, Johnson CE, et al. A centralised telephone service for tobacco cessation: the California experience. Tob Control 2000;9/Suppl 2):ii48-55.

22 McAfee T, Sofian NS, Wilson J, et al. The role of tobacco intervention in population-based health care: a case study. Am J Prev Med 1998;14:46-52.

23 Borland R, Balmford J, Segan C, et al. The effectiveness of personalized smoking cessation strategies for callers to a quitline service. Addiction 2003;98:837-46.

24 Zhu SH, Stretch V, Balabanis M, et al. Telephone counseling for smoking cessation: effects of single-session and multiple-session interventions. J Consult Clin Psychol 1996;64:202-11.

25 Gilbert H, Sutton S. Evaluating the effectiveness of proactive telephone counselling for smoking cessation in a randomized controlled trial. Addiction 2006; 101:590-8.

26 Swan GE, McAfee T, Curry SJ, et al. Effectiveness of bupropion sustained release for smoking cessation in a health care setting: a randomized trial. Arch Intern Med 2003;163:2337-44.

27 Zhu SH, Tedeschi G, Anderson CM, et al. Telephone counseling as adjuvant treatment for nicotine replacement therapy in a "real-world" setting. Prev Med 2000;31:357-63.

28 Macleod ZR, Charles MA, Arnaldi VC, et al. Telephone counselling as an adjunct to nicotine patches in smoking cessation: a randomised controlled trial. Med J Aust 2003;179:349-52.

29 Reid RD, Pipe A, Dafoe WA. Is telephone counselling a useful addition to physician advice and nicotine replacement therapy in helping patients to stop smoking? A randomized controlled trial. CMAJ 1999;160:1577-81.

30 Halpin HA, McMenamin SB, Rideout J, et al. The costs and effectiveness of different benefit designs for treating tobacco dependence: results from a randomized trial. Treating Tobacco Dependence 2006;43:54-65.

31 Solomon L, Scharoun GM, Flynn BS, et al. Free nicotine patches plus proactive telephone peer support to help low-income women stop smoking. Prev Med 2000;31:68-74.

32 Cummings KM, Hyland A, Fix B, et al. Free nicotine patch giveaway program 12-month follow-up of participants. Am J Prev Med 2006;31:181-4.

33 Tunis SR, Stryer DB, Clancy CM. Practical clinical trials: Increasing the value of clinical research for decision making in clinical and health policy. JAMA 2003;290: 1624-32.

34 Rollnick S. What is motivational interviewing? Motivational Interviewing Newsletter for Trainers 1995;2(Issue 3): 1-8.

35 Rollnick S, Miller W. What is motivational interviewing? Behavioral Cognitive Psychotherapy 1995;23:325-34.

36 Miller W, Rollnick S. Motivational interviewing: preparing people to change addictive behavior. New York: Guilford Press, 1991.
37 Schafer JL, Graham JW. Missing data: our view of the state of the art. Psychol Methods 2002;7:147-77.

38 Solas V. 3.0. Cork, Ireland: Statistical Solutions, Ltd. 2001.

39 US Department of Labor. Bureau of Labor Statistics. Available at http:www.bls.gov/opub/mir/1996/12/art6exc.htm.2005.

40 Fix B, Carlin S, Celestino P, et al. Free NRT programs: a report on the reach, efficacy, and cost-effectiveness of NRT give away programs conducted in New York state, 2004.

41 An LC, Schillo BA, Kavanaugh A, et al. Access to nicotine replacement therapy as part of a statewide tobacco telephone helpline. Am J Health Promotion 2006;20:267-71.

42 Miller N, Frieden TR, Liu SY, et al. Effectiveness of a large-scale distribution programme of free nicotine patches: a prospective evaluation. Lancet 2005;365: 1849-54.

43 Swartz SH, Cowan TM, Klayman JE, et al. Use and effectiveness of tobacco telephone counseling and nicotine therapy in Maine. Am J Prev Med 2005;29:288-94.

44 Cummings KM, Fix B, Celestino $P$, et al. Reach, efficacy, and cost-effectiveness of free nicotine medication giveaway programs. J Public Health Manag Pract 2006;12:37-43.

45 Bush T, Zbikowski SM, McClure J, et al. Treatment utilization and adherence in a telephonic tobacco cessation program. Proc National Conference on Tobacco or Health 2003.

46 Hall SM, Delucchi KL, Velicer WF, et al. Statistical analysis of randomized trials in tobacco treatment: Longitudinal designs with dichotomous outcome. Nicotine Tob Res 2001;3:193-202.

47 Glasgow RE, Mullooly JP, Vogt TM, et al. Biochemical validation of smoking status: pros, cons, and data from four low-intensity intervention trials. Addict Behav 1993; 18:511-27

48 Velicer WF, Prochaska JO, Rossi JS, et al. Assessing outcome in smoking cessation studies. Psychol Bull 1992;111:23-41.

49 Patrick DL, Cheadle A, Thompson DC, et al. The validity of self-reported smoking: a review and meta-analysis. Am J Public Health 1994;84:1086-93.

50 SRNT Subcommittee on Biochemical Verification. Biochemical verification of tobacco use and cessation. Nicotine Tob Res 2002;4:149-59.

51 Fu SS, Partin MR, Snyder A, et al. Promoting repeat tobacco dependence treatment: are relapsed smokers interested? Am J Manag Care 2006;12:235-43.

52 McAfee TA. Managed care and the state tobacco settlements. Tob Control 2000;9(Suppl 1):117.

53 Schroeder SA. What to do with a patient who smokes. JAMA 2005;294:482-7. 